\title{
A Tessitura Analítica Bibliométrica da Produção Internacional da COVID-19 no contexto das áreas de Ciências Sociais e Naturais
}

\author{
The Bibliometric Analytical Tessiture of COVID-19 International Production in the context of
}

Social and Natural Sciences areas

\author{
La Tesitura Analítica Bibliométrica de la Producción Internacional de COVID-19 en el contexto de \\ las áreas de Ciencias Sociales y Naturales
}

Recebido: 04/06/2021 | Revisado: 10/06/2021 | Aceito: 11/06/2021 | Publicado: 26/06/2021

\author{
Lúcia Silva Albuquerque de Melo \\ ORCID: https://orcid.org/0000-0002-9543-5642 \\ Universidade Federal de Campina Grande, Brasil \\ E-mail: luciasalbuquerque@gmail.com \\ José Erivaldo da Silva \\ ORCID: https://orcid.org/0000-0001-6682-852X \\ Universidade Federal de Campina Grande, Brasil \\ E-mail: jerysilva85@gmail.com \\ Roberto Roman dos Santos \\ ORCID: https://orcid.org/0000-0003-1003-6237 \\ Universidade Federal de Campina Grande, Brasil \\ E-mail: roberto.roman3004@gmail.com \\ Ângela Maria Cavalcanti Ramalho \\ ORCID: https://orcid.org/0000-0002-8567-4367 \\ Universidade Estadual da Paraíba, Brasil \\ E-mail: profangelauepb@gmail.com
}

\begin{abstract}
Resumo
O artigo tem como objetivo analisar a produção científica internacional sobre o novo coronavírus (COVID-19) na perspectiva das ciências sociais e naturais, visando compreender as características das publicações científicas. Como procedimento metodológico foi realizado uma análise Bibliométrica, por meio de métodos bibliométricos como técnicas avaliativas, técnicas relacionais utilizando o programa VOSviewer. O protocolo de pesquisa consistiu em análise de artigos referentes às temáticas da COVID-19 no contexto das áreas de Ciências sociais e Naturais, a partir da base de dados Web of Science abrangendo o ano de 2020. Foram executadas duas etapas de filtragem. Na primeira optou-se por manter como tipos de documentos apenas artigos científicos no idioma inglês e, na segunda, procedeu-se a inclusão de áreas de pesquisa dentro do escopo da temática em estudo, totalizando, ao final, 379 artigos. A etapa seguinte constituiu-se de avaliação relacionada às áreas de pesquisa, periódicos, países, referências com maior número de citação e estudos mais relevantes do fator $\mathrm{H}$ (H-index). O uso do software VosViewer possibilitou a organização de grupos (cluster) baseados em cocitação e as redes de coocorrências de palavras-chave. Os resultados demonstram em termos avaliativos, que as instituições mais influentes nas pesquisas relacionadas a pandemia da COVID-19 estão concentradas em países como China $(22,9 \%)$ do universo, seguidas por Itália, Estados Unidos, Inglaterra, Canadá, Austrália, Índia, Espanha, Brasil e Alemanha. Os periódicos mais relevantes em termos de publicação, dos 51 periódicos identificados, são: International Journal of Environmental Research and Public Health e Science of the Total Environment, A obra de referência é Immediate Psychological Responses and Associated Factors during the Initial Stage of the 2019 Coronavirus Disease (COVID-19) Epidemic among the General Population in China. O mapa de palavras-chave apresentou o coronavírus como tema central do mapa seguido por termos como surto, confinamento, mortalidade, saúde pública, China, crise, risco, gestão, modelo, desempenho, comportamento, resiliência, incerteza, saúde metal, ansiedade, infecção, conhecimento, depressão, impacto psicológico e estresse. Além disso, também realizou-se uma análise nas obras consideradas referência na rede de citações, destacando os principais propósitos e/ou resultados de cada estudo. Evidenciando, portanto, a emergência e relevância do tema e como este vem sendo explorado.
\end{abstract}

Palavras-chave: Estudo bibliométrico; COVID-19; Ciências sociais; Ciências naturais.

\section{Abstract}

The article aims to analyze the international scientific production of the new coronavirus (COVID-19) from the perspective of social and natural sciences, aiming to understand the characteristics of scientific publications. Bibliometric analysis was performed as a methodological procedure, using bibliometric methods such as evaluative techniques and relational techniques using the VOSviewer program. The research protocol consisted of analyzing 
articles referring to the themes of COVID-19 in the context of the areas of social and Natural Sciences, based on the Web of Science database covering the year 2020. Two filtering steps were performed. In the first, it was decided to keep only scientific articles in English as types of documents and, in the second, the inclusion of research areas within the scope of the theme under study, totalling, in the end, 379 articles. The next stage consisted of an evaluation related to the research areas, journals, countries, references with the highest number of citations and the most relevant studies of the $\mathrm{H}$ factor (H-index). The use of the VosViewer software enabled groups (cluster) based on co-quotation and the co-occurrence networks of keywords. The results demonstrate that the most influential institutions in research related to the COVID-19 pandemic are concentrated in countries such as China (22.9\%) in the universe, followed by Italy, the United States, England, Canada, Australia, India, Spain, Brazil and Germany. The most relevant journals in terms of publication, of the 51 journals identified, are: International Journal of Environmental Research and Public Health and Science of the Total Environment, The reference work is Immediate Psychological Responses and Associated Factors during the Initial Stage of the 2019 Coronavirus Disease (COVID-19) Epidemic among the General Population in China. The keyword map presented coronavirus as the central theme of the map followed by terms such as outbreak, confinement, mortality, public health, China, crisis, risk, management, model, performance, behaviour, resilience, uncertainty, mental health, anxiety, infection, knowledge, depression, psychological impact and stress. In addition, an analysis of the articles considered referenced in the citations network was also performed, highlighting the main objectives and results of each study. Evidencing, therefore, the emergence and relevance of the theme and how it has been explored.

Keywords: Bibliometric study; COVID-19; Social sciences; Natural sciences.

\section{Resumen}

El artículo tiene como objetivo analizar la producción científica internacional sobre el nuevo coronavirus (COVID-19) desde la perspectiva de las ciencias sociales y naturales, con el objetivo de comprender las características de las publicaciones científicas. Como procedimiento metodológico se realizó un análisis bibliométrico, utilizando métodos bibliométricos como técnicas evaluativas, técnicas relacionales utilizando el programa VOSviewer. El protocolo de investigación consistió en analizar artículos referidos a los temas de COVID-19 en el contexto de las áreas de Ciencias Sociales y Naturales, a partir de la base de datos Web of Science del año 2020. Se realizaron dos pasos de filtrado. En el primero, se decidió mantener únicamente los artículos científicos en inglés como tipos de documentos y, en el segundo la inclusión de áreas de investigación dentro del alcance de la temática en estudio, totalizando, al final, 379 artículos. La siguiente etapa consistió en una evaluación relacionada con las áreas de investigación, revistas, países, referencias con mayor número de citas y estudios más relevantes del factor $\mathrm{H}$ (índice $\mathrm{H}$ ). El uso del software VosViewer permitió la organización de grupos (clusters) basados en redes de co-ocurrencia de palabras clave y cocitación. Los resultados demuestran, en términos evaluativos, que las instituciones más influyentes en la investigación relacionada con la pandemia COVID-19 se concentran en países como China (22,9\%) en el universo, seguida de Italia, Estados Unidos, Inglaterra, Canadá, Australia, India, España, Brasil y Alemania. Las revistas más relevantes en términos de publicación, de las 51 revistas identificadas, son: Revista Internacional de Investigación Ambiental y Salud Pública y Ciencia del Medio Ambiente Total, El trabajo de referencia es Respuestas Psicológicas Inmediatas y Factores Asociados durante la Etapa Inicial del Coronavirus 2019 Enfermedad (COVID-19) Epidemia entre la población general en China. El mapa de palabras clave presentó coronavirus como el tema central del mapa seguido de términos como brote, confinamiento, mortalidad, salud pública, China, crisis, riesgo, gestión, modelo, desempeño, comportamiento, resiliencia, incertidumbre, salud del metal, ansiedad, infección, conocimiento, depresión, impacto psicológico y estrés. Además, también se realizó un análisis de los trabajos considerados de referencia en la red de citas, destacando los principales propósitos y / o resultados de cada estudio. Evidenciando, por tanto, la emergencia y relevancia del tema y cómo se ha explorado.

Palabras clave: Estudio bibliométrico; COVID-19; Ciencias sociales; Ciencias naturales.

\section{Introdução}

Em 31 de dezembro de 2019, a Organização Mundial da Saúde (OMS) foi informada sobre diversos casos de pneumonia na cidade de Wuhan, na China. A partir desses dados, foi identificado um novo tipo de coronavírus, que não havia sido detectado em humanos até então, a COVID-19 (SARS-CoV-2) (OPAS/OMS, 2020). Em 11 de março de 2020, a OMS caracteriza a COVID-19 como uma pandemia devido à distribuição geográfica da doença. De acordo com a OMS, em 27 de julho, são mais de 16 milhões de casos confirmados em todo o mundo com 646.641 mil mortes (WHO, 2020).

A origem do novo coronavírus, COVID-19, ainda é incerta, porém a provável fonte de transmissão é um animal, sendo os morcegos os principais candidatos (Zhou et al., 2020). As zoonoses, ou seja, doenças transmitidas de animais para seres humanos, têm graves consequências para o desenvolvimento econômico, o bem-estar animal e humano e a integridade do ecossistema (Grace et al., 2016). 
Com o advento da COVID-19 e a pandemia que surgiu, as pesquisas em busca de uma vacina intensificaram-se rapidamente em escala global, tornando a área de ciências médicas um dos atores principais. Contudo, as ciências sociais também assumiram um protagonismo muito relevante perante à situação, visto que causou impactos significativos nos empregos e na vida cotidiana em geral. As áreas de estudo principais, na área social, fazem referência à formulação de políticas, práticas de isolamento social, controle de fronteiras e auto isolamento (Wen et al., 2020).

A área econômica também sofreu grande impacto com a pandemia, surtindo efeitos negativos no desenvolvimento econômico global, principalmente nos países em desenvolvimento, resultando em uma gama de medidas fiscais, políticas monetárias e econômicas do setor privado entre os vários países ao redor do globo (Sarkodie \& Owusu, 2020). A economia global está passando pela pior recessão desde 1930, com um declínio no PIB de mais de 20\% e um aumento do desemprego, principalmente dos jovens com menos qualificações nos países de média e baixa renda, necessitando de uma política fiscal e monetária adequadas ao momento para auxílio da população (OECD, 2020).

A área ambiental, das ciências naturais, teve consequências vultosas com o surto do novo coronavírus. Com a redução de utilização de transportes e de atividades industriais durante a pandemia, a concentração de gases com emissão antropogênica tem diminuído, o que favorece a saúde de todos e, principalmente, a parte respiratória que é a principal a ser atingida pelo vírus (Wang et al., 2020). Porém, os resíduos hospitalares aumentaram consideravelmente devido à pandemia, o que impacta diretamente o meio ambiente (Sarkodie \& Owusu, 2020).

No atual contexto vivenciado por todo o planeta, o conceito de sociedade de risco do alemão Ulrich Beck (2011) está claramente desenhado na pandemia da COVID-19. Observando a obra do sociólogo alemão e as consequências da pandemia apresentadas, percebe-se cinco pilares de seu pensamento que verificamos na situação presenciada: (a) desenvolvimento de riscos que ameaçam a sociedade em caráter global; (b) globalização como uma sociedade de risco internacional; (c) a opinião de especialistas no centro das tomadas de decisões devido à complexidade e tamanho do risco, deixando governos e indivíduos dependentes de suas visões; (d) individualização da sociedade; e (e) posições de risco: classes e estruturas sociais reduzem, porém a desigualdade permanece (Nygren \& Olofsson, 2020).

Conforme estudo de Vieira et al., (2020), as populações vulneráveis como indígenas, que, aliás, possuem um sistema imunológico diferente, migrantes e refugiados, idosos e famílias numerosas com restrições financeiras sofrem os efeitos mais graves da pandemia, o que corrobora a tese de Beck sobre a sociedade de risco.

Diante do contexto, o presente estudo tem como objetivo principal analisar a produção científica internacional como estudo bibliométrico sobre o novo coronavírus (COVID-19) a partir do enfoque das ciências sociais e naturais e as especificidades.

Acerca das contribuições e relevância alcançadas por esta pesquisa, podem-se citar duas perspectivas: (1) Contribuir para a análise e reflexão das consequências sociais da pandemia da COVID-19, a fim de proporcionar uma visão abrangente do mundo globalizado e seus impactos dentro de uma sociedade de risco; e (2) disponibilizar um panorama das pesquisas científicas relativas às ciências sociais e naturais e suas interações com a COVID-19.

\section{Revisão Teórica}

A sociedade mundial sofre transformações rápidas configurando uma realidade difícil e complexa, com o horizonte da globalização que nos ameaça pelo vírus impiedoso. Constata-se que quase todos os lugares foram alcançados, cada espaço social foi afetado tanto a metrópole como a pequena cidade sob diversos aspectos, a nova realidade muda substancialmente a ideia de espaço e lugar, de espaço, além da rotina sem dinamismo e permeada de preocupações e questionamentos.

Neste sentido, constata-se que a sociedade no campo global está intimamente ligada a uma Pandemia da COVID-19, visto que a dinâmica espaço tempo possibilitou a rápida disseminação do vírus em termos planetário. Observando as condições 
nas quais o vírus tem percorrido, fica evidente que essa dinâmica não representa apenas um fenômeno natural neutro de unificação do mundo possibilitando o progresso, mesmo porque há um viés diretamente relacionado a isso, a circulação de pessoas e objetos, determinado o progresso social do mundo (Souza, 2020).

O processo de determinação social pode ser observado desde a transição do Feudalismo ao Capitalismo, especialmente com as grandes navegações e agregado a isso o progresso técnico que juntos possibilitaram o acúmulo primitivo do capital, especialmente nos países de origem clássica do capitalismo. As identidades sociais são criadas pela determinação social em que são forjadas, em um processo de circulação de bens serviços, mercadorias e pessoas em um ciclo contínuo de globalização, que determina a globalização da saúde, tanto em níveis individuais quanto coletivos, o que de acordo com Berlinguer (1999), esse processo de globalização das doenças tem início em 1492, quando da dominação das Américas por Cristóvão Colombo.

Na busca por novos mercados em um processo contínuo da reprodução da dinâmica e universal do capital, esse processo torna-se particular pelo processo também de circulação social das condições de saúde, isto posto pelo caráter social da reprodução do capital; que analisado globalmente não significa dizer que se está anulando as determinações biológicas da saúde, ou seja, a análise global da movimentação social, leva ao entendimento de que as condições biológicas sejam levadas a outros patamares (Collins; Florian \& Renn, 2020), ou seja, as condições ora propostas determinam condições as quais as características biológicas sejam elevadas ao globo, assim como o risco de morte por microrganismos.

Há um processo universal do ser social, em uma complexidade tal que influencia diretamente no comportamento das pessoas, de modo que mesmo em termos gerais é necessário compreender as particularidades de todo o processo em sua complexidade, visto que as ações individuais são levadas ao coletivo à medida que as relações sociais são postas no processo universal do ser socialmente existente, no caso específico da saúde esse processo não é diferente mesmo porque as ações individuais de uma forma ou de outra socializa-se em saúde coletiva.

As relações sociais historicamente travadas acabam sendo a essência do processo saúde-doença, a partir das relações sociais e não como núcleo imutável, visto que a dinâmica social acaba por promover a dinâmica da saúde biológica e social da humanidade. É essencial entender a partir da premissa da dinâmica social a dinâmica da saúde para não cair em análise fatalista deixando a parte o caráter conjuntural de todo o processo e/ou o que poderia ser de pior situação compreender o processo pela causalidade própria da saúde (Mostafanezhad, 2020).

Compreender a COVID-19 a partir dessas premissas possibilita vincular seu processo de disseminação esteja diretamente ligado com a mundialização do capital, visto que a circulação de bens, serviços, mercadoria e pessoas promovem conjuntamente a circulação do vírus causador da COVID-19, no entanto, não se pode deixar de vincular a esse processo as determinações biológicas específicas para resultar na pandemia anunciada em Março de 2020 pela OMS, uma vez que não é qualquer doença infecciosa que se torna pandemia e/ou transmite-se tão rapidamente sem a combinação de fatores biológicos e sociais.

Conhecendo o vírus e as formas mais comuns de transmissão, as autoridades mundiais adotaram medidas de isolamento, visto que a capacidade de combate às estruturas das unidades de saúde não estava preparada para atender a demanda por leitos, dadas a velocidade da contaminação, impor barreiras sanitárias e as medidas de isolamento social é na verdade uma tentativa de controle do processo de contaminação evitando assim que os hospitais alcancem seus limites rapidamente.

Nesse sentido, pode se dizer que a circulação de pessoas, bens, serviços e mercadorias passou a ser um risco à sociedade, uma vez que esse processo de reprodução do próprio capital é inerente a produção social do risco, e, no processo de distribuição das mercadorias, na circulação das pessoas e de bens tem-se a possibilidade de promoção mais rapidamente a contaminação pelo vírus causador da COVID-19, isto posto que esse processo está intimamente ligado ao próprio processo de 
reprodução da sociedade capitalista que ao mesmo tempo em que produz riqueza distribui riscos a sociedade fator que em tempos de pandemia potencializa o fator de transmissão.

Os riscos produzidos pela sociedade capitalista de produção são resultado de seu próprio processo de produção e reprodução do capital, assim como das condições nas quais a própria sociedade se consolidou, isto desde as condições primitivas da acumulação do capital, nesse processo ao mesmo tempo em que a riqueza é produzida, ocorre a distribuição social do risco produzido no processo de reprodução do capital.

A produção social do risco está diretamente vinculada à produção e reprodução de bens serviços e mercadorias (Williams \& Kayaoglu, 2020), desde os primórdios do capitalismo com a acumulação primitiva do capital, especificamente nos países de produção capitalista tardia. De acordo com Beck (2011, p.23), "a produção social de riqueza é acompanhada sistematicamente pela produção social de riscos" de sorte que os problemas de escassez, está diretamente relacionado à produção, ao mesmo tempo em que há distribuição de riqueza há distribuição de risco.

Em se tratando da pandemia causada pelo novo coronavírus, tem-se alcançado níveis potencialmente maiores de ocorrência de um processo distributivo da riqueza e dos riscos de forma desigual, sendo que a riqueza concentrada com alguns poucos de camadas sociais mais elevadas e os riscos ameaçando a todos independentes da camada social que ocupa, mas ainda assim são os mais pobres os mais vulneráveis a pandemia (Beck, 2011).

Assim sendo, é possível compreender a partir de uma reflexão não necessariamente aprofundada a maneira pela qual a pandemia denúncia e acentua as desigualdades sociais já existentes e marcadas pelo risco especialmente quando se tem fatores sociais que denunciam as desigualdades tanto de acesso quanto de produção tecnológica humana, Beck (2011) compreende, que um sistema de atração do risco pela pobreza.

Como as populações mais pobres precisam mais dos benefícios financeiros do desenvolvimento industrial, parece necessário sublimar o risco, deixando que ele se aproxime, denotando a tese de que "a história da distribuição de riscos mostra que, como a riqueza, os riscos aderem aos padrões de classes, mas inversamente: riqueza acumula no topo, risco na base" (Beck, 2011, p. 41).

Visto de outra forma, a situação difere da vivenciada nos países ricos, que apesar de temerem a contaminação por coronavírus acreditam nas políticas de seus países, uma explicação para isso dar-se pela divulgação na mídia com dados confiáveis e a pouca cobertura para outras doenças, "The COVID-19 has correctly been treated as supremely important, dominating virtually every home page and broadcast" (Troisi, 2020, p.72). No entanto, são os países mais pobres o que tem maior propensão a serem acometidos pelos riscos, especialmente em se tratando dos empregos, isso pelas condições marginais em que ocorrem o desenvolvimento nesses países.

A baixa empregabilidade nas classes mais pobres legitima os efeitos danosos do desenvolvimento (Acselrad, 1996). A recomendação do ficar em casa, não é acompanhada, na mesma proporção, de políticas de compensação financeira ou fiscalização do setor empregatício, o que faz com que as classes menos abastadas tenham de ser obrigadas a se manterem em trabalhos externo, sob risco de contágio, (Penna, 2020).

De acordo Domingues et al., (2020) as famílias mais pobres terão suas rendas afetadas em 20\% a mais do que famílias brasileiras de outras classes sociais pelos efeitos econômicos da pandemia. De modo que estes cidadãos desobedecerão às recomendaç̃oes de isolamento, mantendo-se nas ruas trabalhando e correndo riscos de contágio, a fim de desonerar menos a renda familiar.

Desta forma, atinge-se uma gama maior de indivíduos que mais sofrem com os riscos, isto é, os mais pobres. Para Beck (2011 p. 41) essa é exatamente a lógica da sociedade de risco: "riqueza acumula no topo, risco na base", sendo necessário, para o equilíbrio da pirâmide, que o topo invista na redução dos efeitos danosos (Acselrad, 1996), que atingem os 
grupos menos favorecidos. Os danos causados a essas camadas sociais podem ser comparadas a catástrofes naturais como terremoto, quando as camadas mais pobres são atingidas em todos os sentidos (Grundy-Warr \& Lin, 2020).

Ao se resguardar em casa, os cidadãos das camadas mais pobres minimizam os riscos de contágio pelo coronavírus ao mesmo tempo em que aumenta outros riscos, como ameaça a legitimidade, a propriedade e ao lucro, mas principalmente as condições sociais e ecológicas as quais estão inseridos, visto que quanto mais carentes de recursos mais tende-se a se manter fora das rotas de produção e consumo.

Com o advento da pandemia da COVID-19, os riscos parecem está cada vez mais associados às condições em que as pessoas estão inseridas, para conter a pandemia, algumas medidas foram adotadas. A prática do isolamento social tem causado algumas polêmicas, uma vez que algumas autoridades mostram-se céticas quanto à sua eficácia. O fato é que a maior parte dos tomadores de decisão optou por incentivar essa medida, adotando estratégias de controle da mobilidade da população, como o fechamento de escolas e universidades, do comércio não essencial, e de áreas públicas de lazer etc. Como resultado, grande parte da população brasileira apoiou e aderiu ao movimento do isolamento social com o objetivo de se prevenir da COVID-19 e de colaborar com a atenuação da curva de contágio no país (Bezerra, et. al., 2020).

Ao restringir a circulação das pessoas, e, estas estando em suas residências há claramente um processo de diminuição dos contágios, no entanto, ocorre queda significativa na receita das famílias, de modo que há uma propensão significativa ao aumento da pobreza entre as famílias mais pobres com isso os riscos sociais são elevados consideravelmente não pela contaminação considerando que as políticas de isolamento social são eficazes, mas e principalmente riscos de perda do poder de compra, de voltarem aos postos de trabalhos por exigências dos empregadores e/ou para manter o orçamento ou recuperar parte do orçamento perdido.

São diversos os riscos que a pandemia tem trazido a sociedade, e são inúmeros autores e instituições buscando respostas mediante a pesquisa científica para a pandemia, na tentativa de entender o próprio processo de disseminação do vírus, da análise da eficácia das políticas de combate a pandemia, dos possíveis resultados das políticas e da reestruturação da sociedade pós pandemia, de sorte que entender esse cenário a partir de um estudo bibliométrico torna-se essencial, isto posto pelas características desse tipo de estudo para compreender os esforços sociais, econômicos e pessoais dedicados dar entendimento a sociedade sobre a pandemia.

\section{Metodologia}

Com a finalidade de analisar a produção científica internacional sobre a pandemia do Coronavírus no contexto das áreas de Ciências sociais e Naturais foi realizado uma análise Bibliométrica, por meio de técnicas avaliativas, técnicas relacionais e visualização de técnicas bibliométricas de dados utilizando o software VOSviewer e CitNetExplorer.

A bibliometria é definida como uma abordagem para avaliar e monitorar o progresso de determinadas áreas por meio da triagem de dados, incluindo citações, afiliações de autores, palavras-chave, temas discutidos e métodos empregados para estudos publicados (Koseoglu et al., 2016; Okumus et al., 2019).

O mapeamento científico visa construir mapas bibliométricos que descrevem como áreas específicas, domínios científicos, ou campos de pesquisa são conceitualmente, intelectualmente e socialmente estruturado (Cobo et al., 2011).

Neste estudo, a base de dados utilizada foi a Web of Science (WoS), coleção principal. A WoS permite que pesquisadores acessem artigos científicos de todas as áreas de atuação, oferecendo dados sobre resultados, divulgação, colaboração e impacto de pesquisas (Albort-Morant \& Ribeiro-Soriano, 2016). Os periódicos indexados no WoS têm um fator de impacto associado no Journal Citation Reports (JCR), de modo que os artigos acessados são avaliados por um processo de revisão por pares, certificando o conhecimento gerado (Okumus et al., 2019). 
Para operacionalizar o estudo, foi elaborado um protocolo de pesquisa para o delineamento da pesquisa na base de dados da Web of Science, conforme ilustrado na Figura 1.

Figura 1- Procedimento para delineamento do Protocolo de Pesquisa na Web of Science.

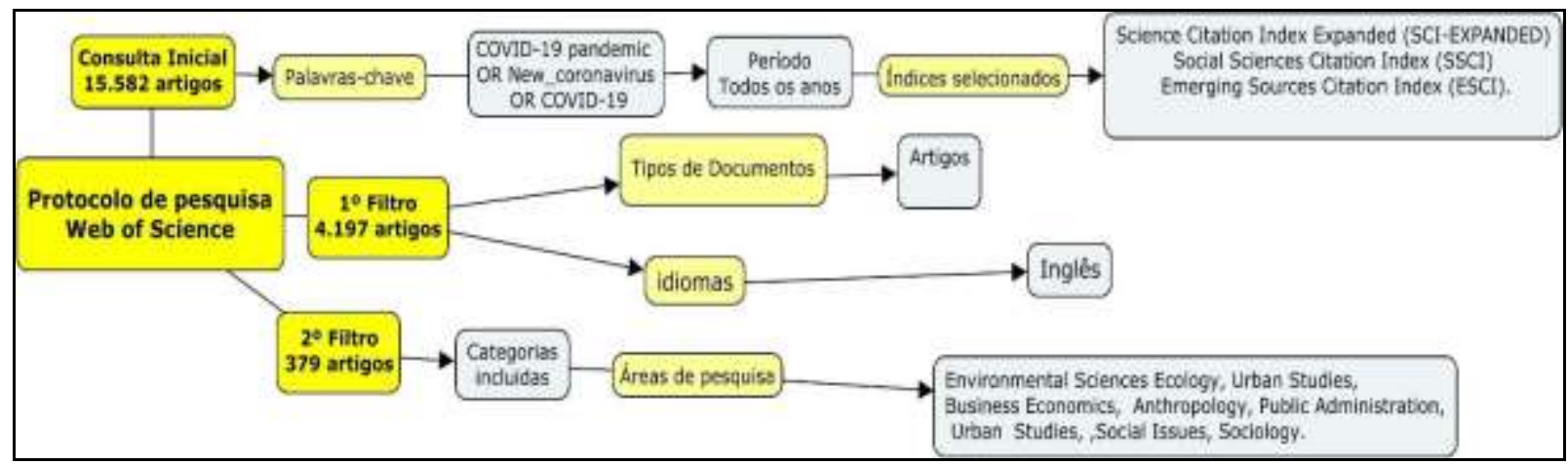

Fonte: Elaborado pelos autores (2020).

A pesquisa na base de dados WoS foi realizada em 25 de julho de 2020. A estratégia de pesquisa tomou como protocolo de busca, a consulta inicial com foco nas palavras-chave "COVID-19 pandemic" OR "New coronavirus" OR "COVID 19", considerou-se os periódicos classificados nos índices: Science Citation Index Expanded (SCI-EXPANDED), Social Sciences Citation Index (SSCI) e Emerging Sources Citation Index (ESCI), porque existem periódicos em ambos os índices que são relacionados a Ciências Naturais e Sociais, o que nos permite expandir a análise abrangendo mais literatura científica no campo de estudo. Utilizou-se como período todos os anos, resultando em um total de 15.582 artigos localizados.

$\mathrm{Na}$ sequência, foram operacionalizados dois filtros de pesquisa. O primeiro filtro, gerou 4.197 artigos tendo como critérios de inclusão no tipo de documento apenas artigos científicos e como idiomas o inglês. Já no segundo filtro, procedeuse a inclusão das áreas de pesquisa: Environmental Sciences Ecology, Business Economics, Urban Studies, Anthropology, Social Issues, Public Administration e Sociology, totalizando, ao final, 379 artigos.

Em seguida, visando a utilização dos softwares VOSviewer e CitNetExplorer, o resultado da pesquisa foi exportado e gravado o conteúdo (registro completo e referências citadas) em um formato de arquivo separado por tabulações win, objetivando-se a utilização dos dados para elaboração das análises bibliométricas.

A primeira etapa dos resultados constitui-se de avaliação relacionada às áreas de pesquisa, periódicos, países, referências com maior número de citação e estudos mais relevantes do fator $\mathrm{H}$ (H-index) por meio do software CitNetExplorer. A segunda etapa utilizou-se o software VOSviewer versão 1.6.12. O VOSviewer é um software direcionado para construção e visualização de redes bibliométricas, analisando uma solução de cluster no nível agregado (Van Eck; Waltman, 2017). O uso do software VosViewer possibilitou a organização de grupos (cluster) baseados em cocitação e as redes de coocorrências de palavras-chave.

\section{Resultados e Discussões}

Os 379 artigos foco deste estudo representam a totalidade de artigos em relação a produção científica internacional sobre o novo coronavírus (COVID-19) a partir do enfoque das ciências sociais e naturais e as especificidades, indexados e publicados na base de dados Web of Science, disponibilizada até a data de consulta realizada em 25 de julho de 2020.

Os resultados do estudo foram evidenciados em três etapas de análise bibliométrica, a primeira descreveu a avaliação relacionada, a partir das categorias utilizadas na Web of Science, como os países onde os artigos foram publicados; áreas de pesquisa, periódicos e obras mais relevantes. Na segunda seção são apresentados e discutidos os resultados do H-index 
extraído do software CitNetExplorer com o ranking de publicações por score de citações, a pesquisa também executou uma análise nos estudos considerados referência na rede de citações, destacando os principais propósitos e/ou resultados de cada estudo. E na terceira etapa da análise bibliométrica foi utilizado o VOSviewer, para a definição das redes com os clusters de coautoria e as palavras-chave com maior relação e frequência nas publicações sobre a temática.

A pesquisa identificou 379 publicações indexadas na base de dados da Web of Science (WoS), disponibilizadas até a data de consulta ( 25 de julho de 2020). A soma do número de citações foi 673, uma média de 1,78 citações por publicação. Cabe ressaltar que todos os 379 artigos pesquisados foram publicados no ano de 2020, este dado é justificado pelo início da pandemia da COVID-19 ter ocorrido mundialmente no início de 2020. Portanto, os estudos nas áreas de Ciências Naturais e Sociais envolvendo a pandemia do novo Coronavírus são recentes, assim como em outras áreas do conhecimento, inclusive na área da saúde.

No tocante às publicações por país, o estudo localizou um total de 64 países que desenvolveram algum tipo de estudo relacionado a pandemia da COVID-19.no contexto das áreas de Ciências Naturais e Sociais. Entre os 10 países com maior número de publicações a China é o país que apresenta o maior número de obras com 87 (22,9\%), na sequência temos a Itália com 62 (16,3\%), Estados Unidos 61 (16\%), Inglaterra 41 (10,8\%), Canadá 26 (6,8\%), Austrália 22 (5,8\%), Índia 22 (5,8\%), Espanha 17 (4,4\%), Brasil 13 (3,4\%) e Alemanha 13 (3,4\%). Em conjunto os 10 países representam mais de 93\% de todas as publicações do campo de estudo.

Com relação às áreas de pesquisa investigadas, a área com mais publicações é a Environmental Sciences Ecology com 243 publicações, representando 64,1 \% do total pesquisado, seguida por Public Environmental Occupational Health, Business Economics, Science Technology Other Topics,Geography, Public Administration, Government Law, Urban Studies, Engineering e Anthropology, constituem as 10 áreas de pesquisa mais representativa do estudo.

No que concerne aos periódicos foram identificados entre as 379 publicações um total de 51 periódicos, a partir disso foram destacados os periódicos mais influentes em termos de números de publicações, sendo o periódico International Journal of Environmental Research and Public Health o mais representativo com 102 (26.9\%) publicações, seguido pelo Science of the Total Environment com 65 (17,1 \%) publicações. Outros que merecem destaque são: Sustainability com 22 (5.8 \%) publicações, Tijdschrift voor Economische en Sociale Geografie (Journal of Economic and Social Geography) com 21 (5,5\%) produções científicas e Tema- Journal of Land Use Mobility and Environment com 19 (5,0 \%) publicações, perfazendo em conjunto um percentual acima de $60 \%$ da produção científica da área em estudo.

Com base nos temas-chave delimitados no estudo e para avaliar com mais propriedade as principais referências, foram identificados os trabalhos que apresentam maior relevância, em termos de publicações, elencados de acordo com seu impacto. Portanto, para evidenciar quais publicações podem ser consideradas referência na rede de citações, utilizou-se o ranking de scores fornecido pelo CitNetExplorer. Com base na listagem de obras, organizadas a partir de ranking de "cit. score", utilizouse o Fator H (H-Index), proposto por Hirsch (2005) para identificar quais autores e, por consequência, obras possuem impacto na área de estudo. Hirsch (2005) propõe que um pesquisador possui Fator H se seu Np (número de publicações) tiver um mínimo de h citações, cada um deles, e, os demais estudos, tiverem menos ou iguais citações cada um. Dessa maneira, foi possível identificar as obras de referência. Para o caso presente, adotou-se h-index $=7$, ou seja, publicações que obtiveram, no mínimo, o valor 7 em seu score, resultando em um total de 7 artigos. Conforme demonstrado no Quadro 1. 
Quadro 1- Publicações de maior impacto -Fator H (H-Index)- CitNetExplorer.

\begin{tabular}{|c|c|c|c|c|c|}
\hline $\mathrm{N}^{\circ}$ & Título & Autores & Título da Fonte & Ano & $\begin{array}{l}\text { Score de } \\
\text { citação }\end{array}$ \\
\hline 1 & $\begin{array}{l}\text { Immediate Psychological Responses } \\
\text { and Associated } \\
\text { FactorsduringtheInitialStageofthe } \\
\text { 2019 CoronavirusDisease (COVID- } \\
\text { 19) Epidemicamongthe General } \\
\text { Population in China. }\end{array}$ & $\begin{array}{l}\text { Wang, Cy; Pan, Ry; } \\
\text { Wan, Xy; Tan, Yl; } \\
\text { Xu, Lk; Ho, Cs; Ho, } \\
\text { Rc }\end{array}$ & $\begin{array}{l}\text { InternationalJournal } \\
\text { of Environmental } \\
\text { ResearchAndPublic } \\
\text { Health }\end{array}$ & 2020 & 20 \\
\hline 2 & $\begin{array}{l}\text { Effectsoftemperaturevariationandhu } \\
\text { midityonthe death of COVID-19 in } \\
\text { Wuhan, China }\end{array}$ & $\begin{array}{l}\text { Ma, Yl; Zhao, Yd; } \\
\text { Liu, Jt; He, Xt; } \\
\text { Wang, B; Fu, Sh; } \\
\text { Yan, J; Niu, Jp; } \\
\text { Zhou, J; Luo, B }\end{array}$ & $\begin{array}{l}\text { Science of The Total } \\
\text { Environment }\end{array}$ & 2020 & 15 \\
\hline 3 & $\begin{array}{l}\text { Correlationbetweenweatherand } \\
\text { COVID-19 pandemic in Jakarta, } \\
\text { Indonesia }\end{array}$ & $\begin{array}{l}\text { Tosepu, R; Gunawan, } \\
\text { J; Effendy, Ds; } \\
\text { Ahmad, Lai; Lestari, } \\
\text { H; Bahar, H; Asfian, } \\
\text { P }\end{array}$ & $\begin{array}{l}\text { Science of The Total } \\
\text { Environment }\end{array}$ & 2020 & 12 \\
\hline 4 & $\begin{array}{l}\text { Severeairpollutioneventsnotavoidedb } \\
\text { yreducedanthropogenicactivitiesduri } \\
\text { ng COVID-19 outbreak. }\end{array}$ & $\begin{array}{l}\text { Wang, Pf; Chen, Ky; } \\
\text { Zhu, Sq; Wang, P; } \\
\text { Zhang, Hl }\end{array}$ & $\begin{array}{l}\text { ResourcesConservati } \\
\text { onandRecycling }\end{array}$ & 2020 & 11 \\
\hline 5 & $\begin{array}{l}\text { Effectofrestrictedemissionsduring } \\
\text { COVID-19 onairquality in India }\end{array}$ & $\begin{array}{l}\text { Sharma, S; Zhang, } \\
\text { My; Anshika; Gao, } \\
\text { Js; Zhang, Hl; Kota, } \\
\text { Sh }\end{array}$ & $\begin{array}{l}\text { Science of The Total } \\
\text { Environment }\end{array}$ & 2020 & 11 \\
\hline 6 & $\begin{array}{l}\text { Associationbetweenambienttemperat } \\
\text { ureand COVID-19 infection in } 122 \\
\text { citiesfrom China }\end{array}$ & Xie, Jg; Zhu, Yj & $\begin{array}{l}\text { Science of The Total } \\
\text { Environment }\end{array}$ & 2020 & 11 \\
\hline 7 & $\begin{array}{l}\text { impactofthe COVID-19 pandemicon } \\
\text { mental healthandqualityoflifeamong } \\
\text { local residents in Liaoningprovince, } \\
\text { china: a cross-sectionalstudy }\end{array}$ & Zhang, Yf; Ma, Zf & $\begin{array}{l}\text { InternationalJournal } \\
\text { of Environmental } \\
\text { ResearchAndPublic } \\
\text { Health }\end{array}$ & 2020 & 7 \\
\hline
\end{tabular}

Fonte: Elaborado pelos autores (2020).

Com esses resultados, realizou-se uma análise nos estudos considerados referência na rede de citações, a partir do Fator $\mathrm{H}$ (H-index), destacando o principal objetivo e/ou resultados de cada estudo.

Verifica-se, no Quadro 1, que a obra com maior impacto é Immediate Psychological Responses and Associated Factors during the Initial Stage of the 2019 Coronavirus Disease (COVID-19) Epidemic among the General Population in China de Wang et al.,(2020), o referido estudo teve como objetivo pesquisar o público em geral na China para entender melhor seus níveis de ansiedade, estresse e depressão durante o estágio inicial do surto de COVID-19, verificando o seu impacto psicológico. A pandemia do coronavírus em 2019 (COVID-19) é uma emergência de saúde pública de interesse internacional e representa um desafio à resiliência psicológica. Os resultados apontaram que 53,8\% dos entrevistados classificaram o impacto psicológico do surto como moderado ou grave, 16,5\% relataram sintomas depressivos moderados a graves, 28,8\% relataram sintomas de ansiedade moderados a graves e 8,1\% relataram níveis de estresse moderado a grave.

O segundo artigo mais citado, 'Effects of temperature variation and humidity on the death of COVID-19 in Wuhan, China". Ma et al., (2020) realizam um estudo com o objetivo de explorar a associação entre as mortes por Coronavírus 
(COVID-19) e os parâmetros climáticos. O estudo, coletou os números de mortes diárias de COVID-19 e parâmetros meteorológicos com dados de poluentes do ar de 20 de janeiro de 2020 a 29 de fevereiro de 2020 em Wuhan na China. A pesquisa, examinou a relação entre fatores meteorológicos e o COVID-19. Os resultados mostraram efeito positivo significativo do Intervalo de Temperatura Diária (Diurnal Temperature Range -DTR) na mortalidade diária de COVID-19, e uma associação negativa significativa entre mortalidade de COVID-19 e temperatura ambiente, bem como umidade absoluta. Os resultados indicam que os efeitos do DTR e da umidade também devem ser observados ao estimar as causas de morte da COVID-19.

O Terceiro estudo mais relevante do h-index é "Correlation between weather and COVID-19 pandemic in Jakarta, Indonesia". Este estudo analisou a correlação entre clima e pandemia de COVID-19 em Jacarta na Indonésia. A pesquisa utilizou uma análise de dados secundários dos dados de vigilância da COVID-19 do Ministério da Saúde da República da Indonésia e do Departamento Meteorológico da República da Indonésia. A pesquisa concluiu que o clima é um fator importante na determinação da taxa de incidência da COVID-19 em Jacarta. A temperatura média foi significativamente correlacionada com o COVID-19 (Tosepu et al., 2020).

No estudo intitulado "Severe air pollution events not avoided by reduced anthropogenic activities during COVID-19 outbreak". Wang et al., (2020) investigaram as influências das reduções de emissões de gases devido à redução das atividades antropogênicas durante o surto de COVID-19 no ar da China. O estudo concluiu que as emissões antropogênicas diminuíram, principalmente, no transporte e na indústria. No entanto, isso não é suficiente para evitar eventos sérios de poluição do ar na maioria das áreas. As taxas de redução nas concentrações de PM2,5 (material particulado fino) foram inferiores às taxas de redução nas emissões precursoras, em parte devido a condições climáticas desfavoráveis. A pesquisa ainda destaca a importância de entender o papel da química e da meteorologia no desenvolvimento de estratégias de controle de emissões.

Sharma et al., (2020) realizou um experimento para verificar a eficácia de emissões restritas na Índia. Devido à pandemia da COVID-19, as atividades humanas são amplamente restritas em muitas regiões da Índia desde de março de 2020. No estudo, foram analisadas concentrações de seis poluentes de critérios, $\mathrm{MP}_{10}, \mathrm{MP}_{2,5}$, dióxido de enxofre $\left(\mathrm{SO}_{2}\right)$, monóxido de carbono (CO), ozônio $\left(\mathrm{O}_{3}\right)$, dióxido de nitrogênio $\left(\mathrm{NO}_{2}\right)$, de 16 de março a 14 de abril de 2017 a 2020 em 22 cidades que cobrem diferentes regiões da Índia.

O sexto estudo é Association between ambient temperature and COVID-19 infection in 122 cities from China. Este estudo teve como objetivo determinar se a temperatura é um fator essencial na infecção causada pelo novo coronavírus. Os resultados indicam que a temperatura média tem uma relação linear positiva com o número de casos de COVID-19 com um limiar de $3{ }^{\circ} \mathrm{C}$. Não há evidências que sustentem que a contagem de casos da COVID-19 possa diminuir quando o clima ficar mais quente, o que fornece implicações úteis para os formuladores de políticas e o público (XIE; ZHU, 2020).

E por fim, o estudo de Zhang e Ma (2020) investigaram o impacto imediato da pandemia de COVID-19 na saúde mental e na qualidade de vida entre residentes chineses locais com mais de 18 anos de idade na província de Liaoning, na China continental. Os autores avaliaram indicadores de impactos negativos na saúde mental, apoio social e familiar, questões relacionadas à saúde mental e mudanças de estilo de vida. A maioria dos participantes $(53,3 \%)$ não se sentiu desamparada devido à pandemia. Por outro lado, $52,1 \%$ dos participantes se sentiram horrorizados e apreensivos devido à pandemia. Além disso, a maioria dos participantes $(57,8-77,9 \%)$ recebeu maior apoio de amigos e familiares, aumentou o sentimento e o carinho compartilhados com familiares e outras pessoas.

$\mathrm{Na}$ análise do mapa bibliométrico de coautoria, por autores, Figura 2, foi considerado a inclusão de todos os autores com alguma publicação no escopo da pesquisa, mesmo que não apresentassem número de citação, inclusive com os autores não conectados. Em virtude da temática de estudo ser recente. A rede completa possui um total de 1.400 autores, universo de publicações pesquisada. Contudo, o software VOSviewer limita à visualização de 1.000 autores, então adotou-se full counting 
do software. Observou-se que muitos destes autores, conforme figura 2, não pertencem fortemente a nenhum cluster. A figura 2 apresenta uma rede muito dispersa com núcleos de autores interligados dos quais apenas alguns possuem conexão com outros clusters. Uma possível justificativa para essa não participação a nenhum clusters é a temática da pandemia da COVID19 se encontra em um estágio inicial de discussão nas diversas áreas do conhecimento, inclusive social e ambiental.

Figura 2 - Mapa Bibliométrico de coautoria, por autores.

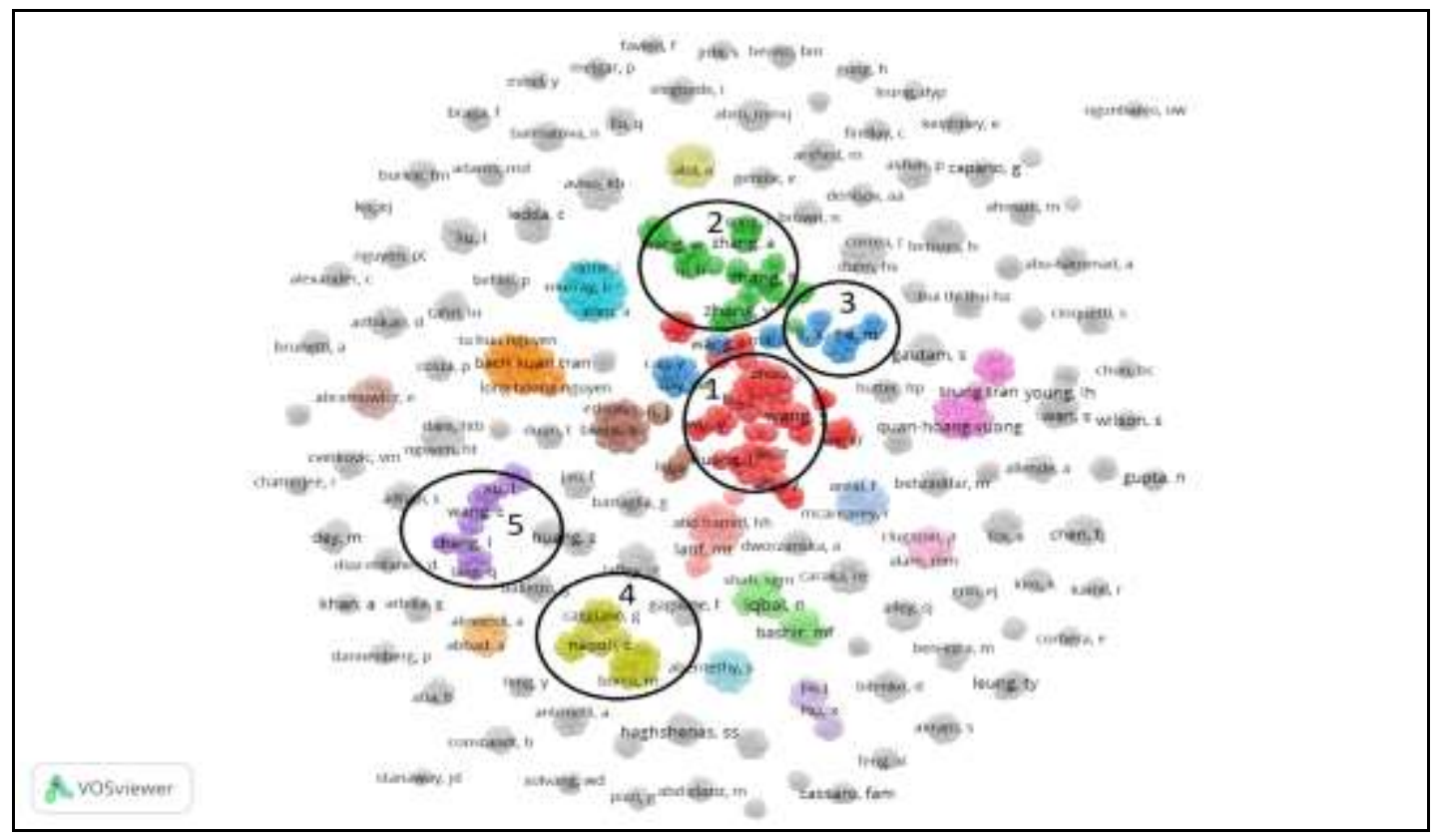

Fonte: Elaborado pelos autores (2020).

Foram identificados 125 clusters tratando da temática em estudo com 4.012 links entre autores. Os cinco (5) principais clusters de cooperação estão destacados com círculo. O principal deles, número 1 em vermelho, é composto por 69 autores, o principal autor no cluster é Zhang, K (14 citações) com 3 publicações e uma relação de força tamanho 42. Na sequência, surge Whang, Y (40 citações) com 6 publicações relação de força tamanho 41. Outros autores de destaque são Li, I (13 citações) e Zhang, X (13 citações) com 2 publicações cada e relação de força tamanho 35 e 34 respectivamente. O segundo cluster (em verde na rede) é composto por 45 autores. O principal autor é Zhang, Y (22 citações) com 6 publicações e uma relação de força na rede de coautoria de 16 .

O terceiro cluster, número 3 em azul, de destaque é composto por 32 autores. A maioria dos autores possui apenas um trabalho publicado, com exceção de Li, X (3 citações) e Wang, I (8 citações) que possuem 3 e 4 publicações respectivamente.

O quarto cluster, em amarelo na rede, possui 30 autores, sendo o Napoli, C (8 citações) o autor principal da rede com publicações e uma relação de força de 31 . No cluster 5, verifica-se o mesmo padrão do cluster 4, ou seja, a grande maioria dos autores apresentam apenas uma publicação e uma relação de força semelhante. A única ressalva é o autor Zhang, I (6 citações) com 3 publicações e relação de força 14. A análise de coautor tem como atributo positivo o fato de que pode proporcionar a colaboração e produção em uma área do conhecimento. Essa análise conecta autores quando eles são coautores em um documento (Zupic \& Cater, 2014).

Contudo, visando uma análise mais acurada, optou-se pela reexecução dos parâmetros anteriores, alterando a exibição final de modo que o mapa fosse construído apenas com o conjunto de autores conectados. 
Figura 3 - Mapa Bibliométrico de coautoria, por autores interligados.

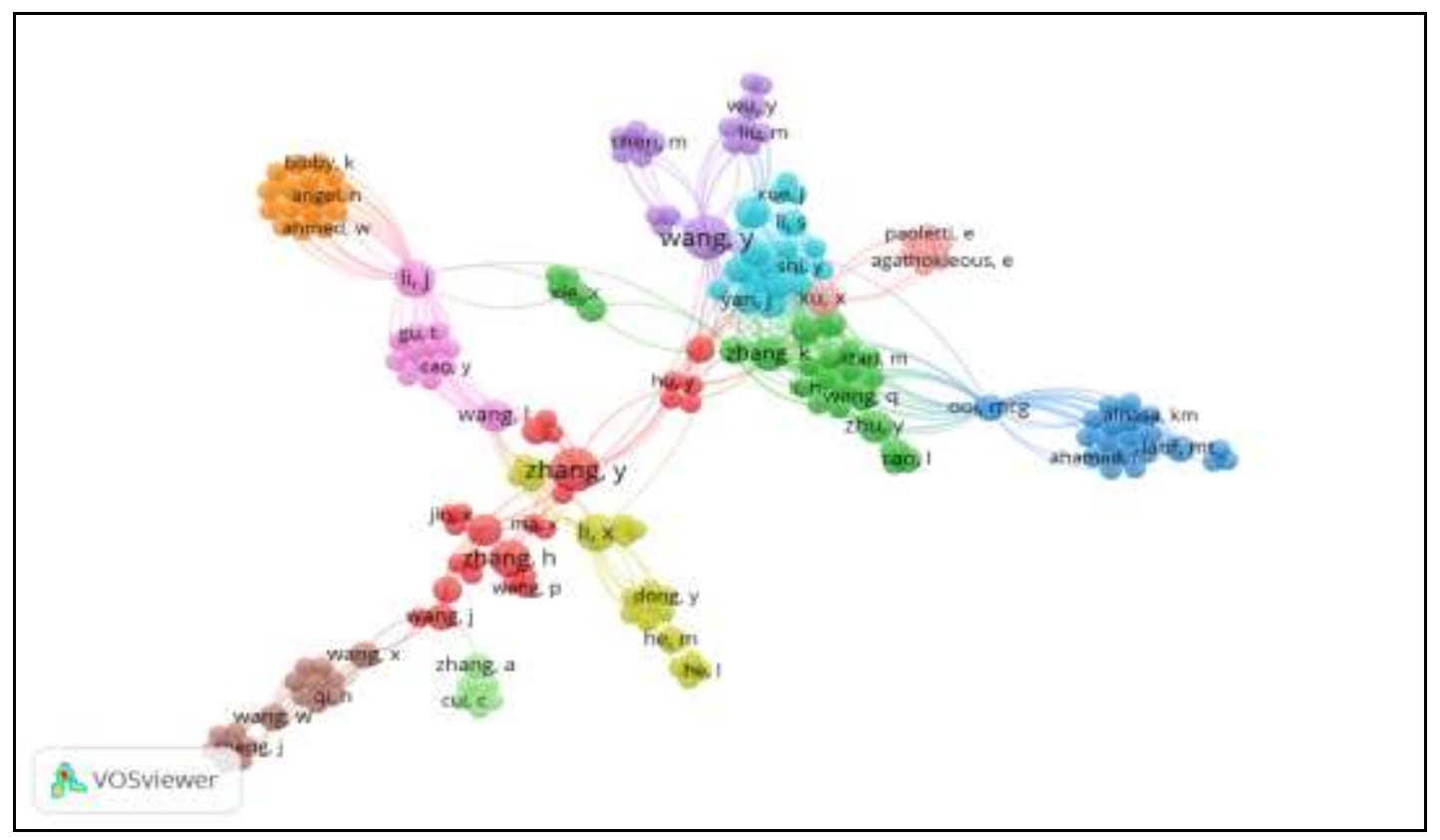

Fonte: Elaborado pelos autores (2020).

A Figura 3 apresenta a rede bibliométrica de coautoria com 189 autores interligados, ou seja, dos 1000 autores apresentados no mapa de coautoria da Figura 2, apenas 189 são autores conectados. Essa rede apresenta poucas ocorrências de itens e apenas clusters 11 conectados.

O mapa de Palavras-Chave (Figura 4) com maior relação e frequência, demonstra a concorrência de palavras dos estudos investigados. A relação de coocorrência entre palavras-chave é estabelecida pelo número de publicações em uma base de documentos em que conecta palavras quando elas aparecem no título, resumo ou palavras-chave. Este método tem como aspecto positivo o fato de usar diretamente o conteúdo dos documentos para análise, ao passo que outras técnicas empregam apenas meta dados bibliográficos (Van Eck \& Waltman, 2014; Zupic \& Cater, 2014).

No estudo foram selecionados termos presentes nas 379 publicações da base de dados. Optando-se pelo método de coocorrência, foram identificadas 1.628 palavras-chave consideradas pelo software, dos quais 41 atendem ao critério de no mínimo 6 ocorrências no texto de acordo com o grau de significância do VOSviewer. Sendo assim, a figura 4 evidencia a formação de três clusters de termos, no qual a palavra-chave COVID-19 está no centro da rede e conectado a todos os clusters, algo esperado e lógico levando em consideração que o objetivo principal da pesquisa era analisar a produção científica internacional como estudo bibliométrico sobre o novo coronavírus (COVID-19) a partir do enfoque das ciências sociais e naturais e as especificidades. 
Figura 4 - Rede de coocorrências de palavra-chave.

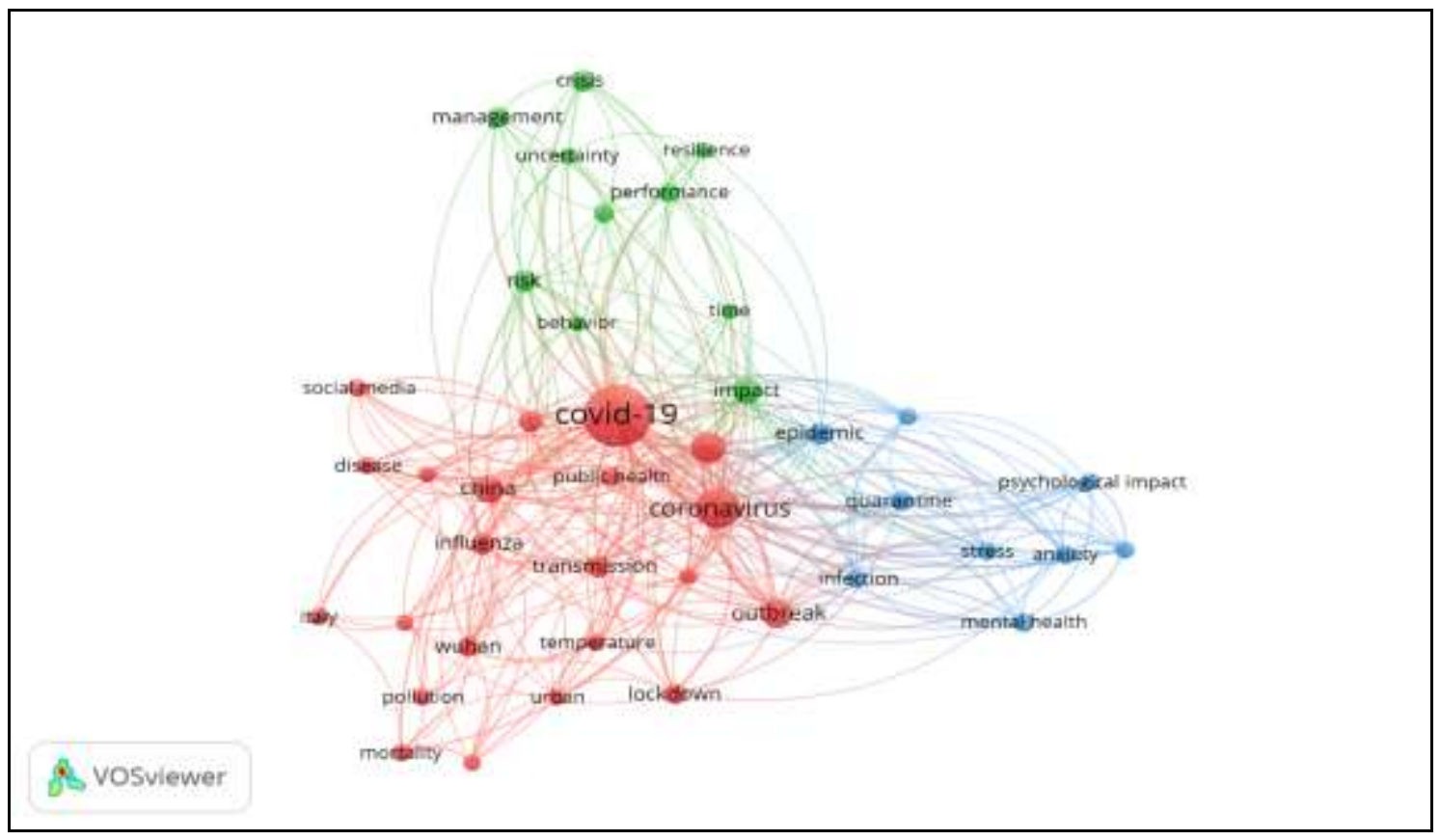

Fonte: Elaborado pelos autores (2020).

O cluster vermelho no mapa é composto por 22 termos (palavras) e sua estruturação também está centrada em torno da COVID-19. Entre os termos presentes, destaca-se os termos com maior frequência de ocorrência: Coronavirus, pandemic (pandemia) outbreak (surto) prevention (prevenção), lockdown (confinamento), mortality (mortalidade), public health (saúde pública), China, information (informações), Air quality (qualidade do ar), air-pollution (poluição do ar) transmission (transmissão), urban (urbano), social media (mídia social) e Wuhan.

O segundo conglomerado em destaque (em verde no mapa) possui 10 termos e é elaborado tendo como termo chave impact (impacto) seguido de crisis (crise), risk (risco), management (gestão), model (modelo), performance (desempenho), behavior (comportamento), resilience (resiliência), uncertainty (incerteza) e time (tempo). Neste cluster é possível observar os elos entre os termos incluídos no conglomerado, e este conjunto sugere pesquisas que abordam os impactos da pandemia sobre os modelos de gestão, as crises causas, comportamento, o risco e incertezas para a sociedade.

O terceiro cluster em destaque (em azul no mapa) apresenta 9 palavras-chaves e tem como elemento mais destacado epidemia. Estão associados aos termos quarentena, saúde mental, ansiedade, infecção, conhecimento, depressão, impacto psicológico e estresse. Neste conjunto, cluster, de termos é possível observar uma discussão sobre a saúde mental neste período de pandemia do COVID-19, seus impactos psicológicos causados pela ansiedade, estresse e depressão.

Os métodos bibliométricos empregam uma abordagem quantitativa para a descrição, avaliação e monitoramento das pesquisas publicadas. Esses métodos têm o potencial de introduzir um processo de revisão sistemática, transparente e com possibilidade de reprodução de pesquisas, melhorando, como isso, a qualidade das revisões. Os métodos bibliométricos são uma ajuda útil nas revisões de literatura mesmo antes do início da leitura, guiando o pesquisador para os trabalhos mais influentes e mapeando o campo de pesquisa sem viés subjetivo (Zupic \& Čater, 2015).

\section{Conclusões}

O presente estudo teve como objetivo principal analisar a produção científica internacional como estudo bibliométrico sobre o novo coronavírus (COVID-19) a partir do enfoque das ciências sociais e naturais e as especificidades. Para isso, realizou um estudo bibliométrico utilizando as técnicas avaliativas e relacionais. E para operacionalizar a pesquisa usou como 
referência as publicações que compõem a base de dados Web of Science. Tendo um universo de 379 publicações selecionadas e analisadas no estudo.

Em termos avaliativos, os resultados demonstram que as instituições mais influentes nas pesquisas relacionadas a pandemia do COVID-19 no contexto das áreas de Ciências Naturais e Sociais estão concentradas em países como China, com maior número de obras, representando 22,9\% do universo, Itália, Estados Unidos, Inglaterra, Canadá, Austrália, Índia, Espanha, Brasil e Alemanha sendo estes destaques na produção sobre a temática. No tocante aos periódicos mais relevantes em termos de publicação, dos 51 periódicos identificados, os cinco principais são: International Journal of Environmental Research and Public Health, Science of the Total Environment, Sustainability Tijdschriftvo or Economis cheen Sociale Geografie (Journalof Economic and Social Geography) e TeMA- Journal of Land Use Mobility and Environment que representam acima de $60 \%$ da produção científica da área pesquisada.

Com relação às obras de referência foi identificado um H-index 11 dentro da base da Web of Science e um H-index 7 (Quadro 2) no software CitNetExplorer. Entretanto, a principal obra permanece sendo a de Wang et al.,(2020), com a publicação Immediate Psychological Responses and Associated Factors during the Initial Stage of the 2019 Coronavirus Disease (COVID-19) Epidemic among the General Population in China.

No que se refere a análise relacional, utilizando o software VosViewer, permitiu, através da elaboração de redes bibliométricas, concluir quais são os principais clusters de coautoria, entre autores, no qual o principal deles, Cluster em vermelho, é composto por 69 autores e tem Zhang, K como principal autor, seguido por Whang, Y. Já na rede de coocorrência de palavras-chave foram identificados 3 clusters, tendo a pandemia do coronavírus como tema central do mapa seguido por termos como surto, confinamento, mortalidade, saúde pública, China, informações no primeiro cluster. O segundo cluster, verde no mapa, foi possível identificar elos entre os termos chaves impacto, crise, risco, gestão, modelo, desempenho, comportamento, resiliência, incerteza e tempo, sugerindo pesquisas que abordam os impactos da pandemia sobre os modelos de gestão, as crises causas, comportamento, o risco e incertezas para a sociedade. E o terceiro cluster possível observar uma discussão sobre a saúde mental neste período de pandemia do COVID-19, seus impactos psicológicos causados pela ansiedade, estresse e depressão.

Em suma, esta pesquisa ofereceu uma contribuição, em âmbito mais generalístico, da produção científica internacional sobre o novo coronavírus (COVID-19) a partir do enfoque das ciências sociais e naturais e as especificidades, ao demonstrar às áreas de pesquisa, periódicos, países, agrupar publicações com base em suas relações de citação em rede de citações com os estudos mais relevantes, demonstrar os cluster com as publicações mais citadas e o ranking de publicações por score de citações. Além disso, também se realizou uma análise nas obras consideradas referência na rede de citações, destacando os principais propósitos e/ou resultados de cada estudo. Evidenciando, portanto, a emergência e relevância do tema e como este vem sendo explorado.

Como sugestão para futuras pesquisas, utilizando a análise bibliométrica como base de estudo, indica-se o uso de outras bases de dados, tais como Scopus, Science Direct, EBSCO, Scielo e periódicos específicos. Considerando as características desta pesquisa, destaca-se que o estudo se restringiu aos dados da base Web of Science, coleção completa, por apresentar compatibilidade de uso com o software CitNetExplorer.

\section{Referências}

Acselrad, H. (1996). Sustainability and Territory. In Internacional Seminar on “Sustainability as a Concept for Social Sciences”. Frankfurt: ISOE/Unesco.

Albort-Morant, G., \& Ribeiro-Soriano, D. (2016). A bibliometric analysis of international impact of business incubators. Journal of Business Research, 69(5), 1775-1779. https://doi.org/10.1016/j.jbusres.2015.10.054

Beck, U. (2011). Sociedade de risco: rumo a uma outra modernidade. Editora 34. 
Berlinguer, G. (1999). Globalização e saúde global. Estudos avançados, 13(35), 21-38. https://doi.org/10.1590/S0103-40141999000100003

Bezerra, A. C. V., Silva, C. E. M. D., Soares, F. R. G., \& Silva, J. A. M. D. (2020). Fatores associados ao comportamento da população durante o isolamento social na pandemia de COVID-19. Ciência \& Saúde Coletiva, 25, 2411-2421. https://doi.org/10.1590/1413-81232020256.1.10792020

Cobo, M. J., López-Herrera, A. G., Herrera-Viedma, E., \& Herrera, F. (2011). Science mapping software tools: Review, analysis, and cooperative study among tools. Journal of the American Society for information Science and Technology, 62(7), 1382-1402. https://doi.org/10.1002/asi.21525

Collins, A., Florin, M. V., \& Renn, O. (2020). COVID-19 risk governance: drivers, responses and lessons to be learned. Journal of Risk Research, 23(7-8), 1073-1082. https://doi.org/10.1080/13669877.2020.1760332

Domingues, E., Freire Cardoso, D., \& Magalhães, A. (2020). Efeitos econômicos negativos da crise do Corona Vírus tendem a afetar mais a renda dos mais pobres (No. 003). Cedeplar, Universidade Federal de Minas Gerais.

Grace, D., Bett, B. K., Lee, H. S., \& MacMillan, S. (2016). Zoonoses: Blurred lines of emergent disease and ecosystem health. United Nations Environment Programme.

Grundy-Warr, C., \& Lin, S. (2020). COVID-19 geopolitics: silence and erasure in Cambodia and Myanmar in times of pandemic. Eurasian Geography and Economics, 61(4-5), 493-510. https://doi.org/10.1080/15387216.2020.1780928

Hirsch, J. E. (2005). An index to quantify an individual's scientific research output. Proceedings of the National academy of Sciences, 102(46), 16569-16572. https://doi.org/10.1073/pnas.0507655102

Koseoglu, M. A., Rahimi, R., Okumus, F., \& Liu, J. (2016). Bibliometric studies in tourism. Annals of Tourism Research, 61, 180-198. https://doi.org/10.1016/j.annals.2016.10.006

Ma, Y., Zhao, Y., Liu, J., He, X., Wang, B., Fu, S., \& Luo, B. (2020). Effects of temperature variation and humidity on the death of COVID-19 in Wuhan, China. Science of the total environment, 724, 138-226. https://doi.org/10.1016/j.scitotenv.2020.138226

Mostafanezhad, M. (2020). COVID-19 is an unnatural disaster: Hope in revelatory moments of crisis. Tourism Geographies, 22(3), 639-645. https://doi.org/10.1080/14616688.2020.1763446

Nygren, K. G., \& Olofsson, A. (2020). Managing the COVID-19 pandemic through individual responsibility: the consequences of a world risk society and enhanced ethopolitics. Journal of Risk Research, 23(7-8), 1031-1035. https://doi.org/10.1080/13669877.2020.1756382

Okumus, F., Köseoglu, M. A., Putra, E. D., Dogan, I. C., \& Yildiz, M. (2019). A bibliometric analysis of lodging-context research from 1990 to 2016. Journal of Hospitality \& Tourism Research, 43(2), 210-225. https://doi.org/10.1177/1096348018765321

OECD (2020), OECD Economic Outlook, 2020 (1), OECD Publishing, Paris.

OPAS/OMS. (2020). Histórico - COVID-19. https://www.paho.org/pt/covid19/historico-da-pandemia-COVID-19

Sarkodie, S. A., \& Owusu, P. A. (2020). Global assessment of environment, health and economic impact of the novel coronavirus (COVID-19). Environment, Development and Sustainability, 1-11. https://doi.org/10.1007/s10668-020-00801-2

Sharma, S., Zhang, M., Gao, J., Zhang, H., \& Kota, S. H. (2020). Effect of restricted emissions during COVID-19 on air quality in India. Science of the Total Environment, 728, 138878. https://doi.org/10.1016/j.scitotenv.2020.138878

Souza, D. D. O. (2020). A pandemia de COVID-19 para além das Ciências da Saúde: reflexões sobre sua determinação social. Ciência \& Saúde Coletiva, 25, 2469-2477. https://doi.org/10.1590/1413-81232020256.1.11532020

Tosepu, R., Gunawan, J., Effendy, D. S., Lestari, H., Bahar, H., \& Asfian, P. (2020). Correlation between weather and COVID-19 pandemic in Jakarta, Indonesia. Science of The Total Environment, 725, 138436. https://doi.org/10.1016/j.scitotenv.2020.138436

Troisi, A. (2020). Fear of COVID-19: insights from evolutionary behavioral science. Clinical Neuropsychiatry, 17( 2), 72-75.

Van Eck, N. J., \& Waltman, L. (2014). CitNetExplorer: A new software tool for analyzing and visualizing citation networks. Journal of informetrics, 8(4), 802-823. https://doi.org/10.1016/j.joi.2014.07.006

Van Eck, N. J., \& Waltman, L. (2017). Citation-based clustering of publications using CitNetExplorer and VOSviewer. Scientometrics, 111(2), 1053-1070. https://doi.org/10.1007/s11192-017-2300-7

Vieira, C. M., Franco, O. H., Restrepo, C. G., \& Abel, T. (2020). COVID-19: The forgotten priorities of the pandemic. Maturitas, 136, 38-41. https://doi.org/10.1016/j.maturitas.2020.04.004

Wang, C., Pan, R., Wan, X., Tan, Y., Xu, L., Ho, C. S., \& Ho, R. C. (2020). Immediate psychological responses and associated factors during the initial stage of the 2019 coronavirus disease (COVID-19) epidemic among the general population in China. International journal of environmental research and public health, 17(5), 1729. https://doi.org/10.3390/ijerph17051729

Wang, P., Chen, K., Zhu, S., Wang, P., \& Zhang, H. (2020). Severe air pollution events not avoided by reduced anthropogenic activities during COVID-19 outbreak. Resources, Conservation and Recycling, 158, 104814. https://doi.org/10.1016/j.resconrec.2020.104814

Wen, J., Wang, W., Kozak, M., Liu, X., \& Hou, H. (2020). Many brains are better than one: The importance of interdisciplinary studies on COVID-19 in and beyond tourism. Tourism Recreation Research, 1-4. https://doi.org/10.1080/02508281.2020.1761120

World Health Organization (WHO). (2020) Coronavirus disease 2019 (COVID-19). Situation Report. 189. Genebra. 
Research, Society and Development, v. 10, n. 7, e39810716822, 2021

(CC BY 4.0) | ISSN 2525-3409 | DOI: http://dx.doi.org/10.33448/rsd-v10i7.16822

Williams, C. C., \& Kayaoglu, A. (2020). The coronavirus pandemic and Europe's undeclared economy: impacts and a policy proposal. South East European Journal of Economics and Business, 15(1), 80-92. https://doi.org/10.2478/jeb-2020-0007

Xie, J., \& Zhu, Y. (2020). Association between ambient temperature and COVID-19 infection in 122 cities from China. Science of the Total Environment, 724 138201. https://doi.org/10.1016/j.scitotenv.2020.138201

Zhang, Y., \& Ma, Z. F. (2020). Impact of the COVID-19 pandemic on mental health and quality of life among local residents in Liaoning Province, China: A cross-sectional study. International journal of environmental research and public health, 17(7), 2381. https://doi.org/10.3390/ijerph17072381

Zupic, I., \& Čater, T. (2015). Bibliometric methods in management and organization. Organizational Research Methods, 18(3), 429-472. https://doi.org/10.1177/1094428114562629

Zhou, P., Yang, X. L., Wang, X. G., Hu, B., Zhang, L., Zhang, W., \& Shi, Z. L. (2020). A pneumonia outbreak associated with a new coronavirus of probable bat origin. nature, 579(7798), 270-273. https://doi.org/10.1038/s41586-020-2012-7 